\title{
Parasites from the fish Clarias gariepinus as potential biondicators for heavy metal pollution in freshwater systems
}

Beaven Utete and Nyasha Mabika

\section{This article has been removed for legal reasons}

